\title{
Antimicrobial and Wound Healing Activity of Ixora coccinea Leaf Extract in Hydrogel Formulation
}

Angeli Ann S. Rescober, Irish Florain M. Blanco, John Albert T. Janio, Shanniel Eccole A. Custodio, Faye Louise D. Daguman, John Matthew D. Viray and Janine H. Dollesin

Adamson University, Manila, Philippines

\begin{abstract}
Objectives. The purpose of this study was to evaluate the antimicrobial and wound healing property of the preformulated hydrogel containing the methanolic leaf extract of Ixora coccinea, as well as its acute dermal irritation using New Zealand rabbits.

Methods. Mature dried leaves of I. coccinea was subjected to extraction using maceration and was concentrated in vacuo. Sodium carboxymethylcellulose and gelatin were used to create hydrogel in which the crude extract was incorporated. Physicochemical properties of the extract and preformulated hydrogel were characterized, while its antimicrobial activities against Pseudomonas aeruginosa, Staphylococcus aureus, and Staphylococcus epidermidis were determined using the agar well method and compared to the standard drug $2 \% \mathrm{w} / \mathrm{v}$ mupirocin ointment. A wound excision model in rats was used to determine the wound healing property of the preformulated hydrogel against povidone-iodine ointment. Lastly, animal testing was performed following the OECD Guidelines and upon approval of the IACUC Committee.
\end{abstract}

Result. The preformulated hydrogel was effective against $S$. aureus ( $p$-value $\leq 0.001$ ) but resistant to $P$. aeruginosa and S. epidermidis. Furthermore, the wound contraction rate in groups treated with preformulated hydrogel $(p$-value $=0.006)$ is significantly higher than in groups treated with hydrogel base and povidone-iodine. Moreover, no dermal erythema and edema were observed with albino rabbits.

Conclusion. The preformulated hydrogel with I. coccinea methanolic leaf extract is non-irritating, effective against staphylococcal infections commonly found in wounds. Hence, it is a good substitute for povidone-iodine in wound treatment.

Key Words: antimicrobial, hydrogel, Ixora coccinea, skin irritation, wound healing

\section{INTRODUCTION}

Best Prize for Oral Presentation. Paper presented in the $7^{\text {th }}$ International Conference on Advanced Pharmaceutical Research (Virtual Conference) on November 5-6, 2020, at Rangsit University, Thailand.

Paper presented in the $4^{\text {th }}$ International Conference on Pharmacy and Pharmaceutical Science (ICPPS) on July 18-19, 2019, at Ajou University, Suwon Korea.

Corresponding author: Angeli Ann S. Rescober Adamson University, Manila, Philippines

Email: angeli.ann.rescober@adamson.edu.ph
Ixora coccinea L., locally known as Santan of the Family Rubiaceae, is a small-medium evergreen shrub widely cultivated throughout Southeast Asian regions and beneficial for treating various ailments such as chronic ulcer, hypertension, menstrual irregularities, sprain, and skin infections. ${ }^{1}$ Its leaf extract is proven effective against Staphylococcus aureus ${ }^{2}$ while its methanolic leaf extracts are effective antioxidants, anti-inflammatory agent, fibroblast proliferative, and stimulates Smad mediated collagen production at $2.5 \%$ with wound contraction rate greater than gentamicin sulfate $(0.01 \% \mathrm{w} / \mathrm{w})$ in both in vitro and in vivo studies. Phytochemical screening showed the presence of alkaloids, flavonoids, tannins, and triterpenes which possibly contribute to its pharmacologic effects., ${ }^{1,3}$

Hydrogels are soft, elastic semisolid dosage forms with $70-90 \%$ water content, easier to be applied on 
wounds, and removed without any skin damages because it provides a moist environment that favors tissue epithelium and granulation which are important wound healing processes. ${ }^{4,5}$ It is ideal for biomedical applications such as the superabsorbent crosslinked hydrogels with higher carboxymethylcellulose (CMC) amounts which swells readily upon liquid contact and enables higher drug loading percentage due to the presence of carboxyl group. ${ }^{6}$ It is widely used in tissue engineering, plant breeding, drug delivery and wound dressing because of its biocompatibility, biodegradability, and wound healing promotion by regulating the transepidermal water movement. ${ }^{7-9}$

This study aimed to utilize $2.5 \%$ crude methanolic leaf extract of $I$. coccinea in preformulating a topical hydrogel and determine its antimicrobial, wound healing property, and acute dermal irritancy for possible wound treatment substitute.

\section{MATERIALS AND METHODS}

\section{Materials}

All materials and chemical reagents were supplied by the Pharmacy Laboratory, Adamson University - College of Pharmacy except for the microorganisms such as Staphylococcus aureus (ATCC 25923), Staphylococcus epidermidis (ATCC 12228), and Pseudomonas aeruginosa (ATCC 27853) which were procured from the Department of Medical Microbiology, College of Public Health, University of the Philippines - Manila.

\section{Collection and Preparation of Extract}

The leaves of I. coccinea were harvested from Laguna during September 2018 in which some fresh plant samples were sent to the Bureau of Plant Industry (BPI) for authentication, while others were washed with tap water to remove dust or any substances that may contaminate the collected samples. It was then air dried, ground using a Holz portable mechanical grinder, macerated with methanol for three days, filtered, concentrated using IKA ${ }^{\circledR}$ rotary evaporator set at $70^{\circ} \mathrm{C}$, and subjected to oven-drying at $45^{\circ} \mathrm{C}$ for 5 hours to evaporate any methanol present and remove moisture from the samples.

\section{Physicochemical Tests for Extract}

a) Physical Appearance - The color and odor of concentrated methanolic leaf extracts of I. coccinea was assessed using visual-tactile method.

b) $\mathbf{p H}$ - Using a $\mathrm{pH}$ meter (Sartorius ${ }^{\circledR}$ Basic Meter PB11), the $\mathrm{pH}$ of concentrated methanolic leaf extract of $I$. coccinea was measured at room temperature in which its reading was recorded after immersing the electrode in the solution.

c) Solubility - The solubility of concentrated methanolic leaf extracts of $I$. coccinea was determined by dissolving one gram of the extract in 10 milliliters $(\mathrm{mL})$ of water, ether, and chloroform.

\section{Hydrogel Preformulation}

Formulation parameters of A. A Rescober (2019) was adapted with some modifications. Carboxmethylcellulose (CMC) was gradually dissolved in $20 \mathrm{~mL}$ deionized water and gelatin in $20 \mathrm{~mL}$ hot deionized water. Once dissolved, the gelatin solution was gradually added to CMC solution and continuously stirred until it became a homogeneous mixture. The methanolic leaf extracts of I. coccinea was dissolved in $20 \mathrm{~mL}$ deionized water then gradually added to the CMCGelatin solution with continuous stirring. A sufficient volume of solvent was added to the mixture to achieve the desired $100 \mathrm{~mL}$ volume. Table 1 below shows the percentage of each excipient and the corresponding use in the preformulation.

Table 1. Hydrogel preformulation

\begin{tabular}{lcc}
\multicolumn{1}{c}{ Ingredients } & Concentration & Uses \\
\hline $\begin{array}{l}\text { l. coccinea methanolic } \\
\text { leaf extracts }\end{array}$ & $2.50 \%$ & Active ingredient \\
Carboxymethylcellulose & $1.60 \%$ & Viscosity enhancer \\
Gelatin & $0.40 \%$ & Viscosity enhancer \\
Deionized water & $95.50 \%$ & Solvent \\
\hline
\end{tabular}

\section{Quality Control Tests for Preformulated Hydrogel}

a) Physical appearance - Color, odor, grittiness, and homogeneity of the preformulated hydrogel were visually analyzed and recorded.

b) $\mathbf{p H}$ - Using a pH meter (Sartorius ${ }^{\circledR}$ Basic Meter PB-11), the $\mathrm{pH}$ of preformulated hydrogel was measured at room temperature in which its reading was recorded after immersing the electrode.

c) Viscosity - SNB-2 Digital Viscometer with spindle No. 4 was used to determine the viscosity of the preformulated hydrogel.

d) Spreadability - The preformulated hydrogel was placed in between two glass slides and slipped off in which the spreadability $(\mathrm{S})$ was calculated using the formula: $S=\frac{M * L}{T}$ wherein $\mathrm{M}$ is the weight of substance placed on the top glass slide, $\mathrm{L}$ is the length traveled and $\mathrm{T}$ is the time taken to separate the two glass slides.

e) Sterility Test - Sodium Thioglycollate (FTM) and Soybean Casein Digest (SCD) media were prepared separately then two milliliters of preformulated hydrogel were transferred using a sterile syringe to each media followed by incubation for 14 days $\left(30-35^{\circ} \mathrm{C}\right.$ for FTM and $20-25^{\circ} \mathrm{C}$ for SCD) in which absence of turbidity indicates sterility of the preformulated hydrogel.

\section{In-vitro Antimicrobial Activity}

The Agar well method was used to determine the antimicrobial activities of preformulated hydrogel wherein an aliquot of $0.5 \mathrm{~mL}$ inoculums of $S$. aureus (ATCC 25923), $S$. epidermidis (ATCC 12228) and P. aeruginosa (ATCC 27853) were spread into separate Petri dishes containing MuellerHinton Agar. It was left for several minutes inside the Class II Biosafety Cabinet (Telstar BIO II Advance 4), punched 
aseptically using disposable $3 \mathrm{~mL}$ sterile syringe barrels, and filled with the following test groups: $100 \mu \mathrm{L}$ hydrogel base (negative control), $100 \mu \mathrm{L}$ Mupirocin Ointment 2\% $\mathrm{w} / \mathrm{v}$ (positive control), and $100 \mu \mathrm{L}$ preformulated hydrogel. The plates were placed inside the incubator for 24 hours at $37^{\circ} \mathrm{C}$ wherein zones of inhibition were measured using a digital Vernier caliper (VINCA DCLA-0605). Results were interpreted based on the 2018 Guidelines of Clinical and Laboratory Standards Institute (CLSI) ${ }^{10}$ as shown in Table 2 below.

Table 2. Interpretative table of selected microorganism for susceptibility testing on Mupirocin based on zone diameter in millimeter $(\mathrm{mm})$

\begin{tabular}{lccc}
\multicolumn{1}{c}{ Microorganisms } & Resistant & Intermediate & Susceptible \\
Staphylococcus spp. & $\leq 28$ & - & $\geq 29$ \\
P. aeruginosa & $\leq 14$ & $15-20$ & $\geq 21$ \\
\hline
\end{tabular}

\section{In vivo Wound Healing Activity and Acute Dermal Irritancy}

\section{Preparation of test animals}

An approval from the Institutional Animal Care and Use Committee (IACUC) of Adamson University was obtained before the conduct of animal testing. Healthy male Sprague Dawley rats at 3 months old weighing $100-200 \mathrm{~g}$ and New Zealand rabbits at $1-2 \mathrm{~kg}$ were purchased from a BAI-Accredited animal breeder (LAF-0036). These test animals were housed inside the Animal House of Adamson University - College of Pharmacy with a maintained temperature of $25 \pm 5^{\circ} \mathrm{C}$ and acclimatized for seven days with free access to food pellets and water ad libitum before the experimental procedures were carried out. Also, these test animals were randomly selected and assigned to different treatment groups to avoid bias.

\section{Wound healing activity}

Acclimatized rats were anesthetized with $10 \mathrm{mg} / \mathrm{kg}$ ketamine intraperitoneally, back hairs were shaved using a razor blade, and the surgical site was disinfected with $70 \%$ alcohol. ${ }^{11}$ The dorsal skin was folded, raised cranially, and caudally at the midline to form a sandwiched skinfold. Then, test animals were placed in a lateral position followed by pressing down with 4-mm diameter sterile biopsy punch to completely removed the two skin layers to create a symmetrical full-thickness excisional wound. ${ }^{12}$ Homeostasis in the wounded area was achieved by blotting the wound with a cotton swab soaked in normal saline solution. As presented in Table 3, test animals were randomly divided into four groups namely the untreated (Group I), treated with hydrogel base (Group II), treated with preformulated hydrogel (Group III), and treated with the standard drug Povidone-Iodine (Group IV). Wounds were left uncovered and rats were then caged individually. ${ }^{11}$
Table 3. Animal Groupings for Wound Excision Model

\begin{tabular}{ccc}
$\begin{array}{c}\text { Treatment } \\
\text { Groups }\end{array}$ & Description & $\begin{array}{c}\text { No. of Rats } \\
\text { Used }\end{array}$ \\
\hline 1 & Untreated & 5 \\
2 & Hydrogel base & 5 \\
3 & Preformulated hydrogel of I. coccinea & 5 \\
4 & Povidone lodine & 5 \\
\hline
\end{tabular}

\section{Wound Area Measurement}

The wound reduction area was monitored on predetermined days $\left(2^{\text {nd }}, 4^{\text {th }}, 8^{\text {th }}, 12^{\text {th }}\right.$, and $16^{\text {th }}$ day $)$ and measured using a Vernier caliper. ${ }^{13}$

\section{Wound Contraction Rate}

Wound Contraction Rate (WCR) was calculated using the formula provided wherein it is the percentage of the reduction in original wound area size as measured using a Vernier caliper in millimeters. ${ }^{13}$

$$
\mathrm{WCR}=\left(\frac{\text { Initial wound area }-\mathrm{N}^{\text {th }} \text { day wound area }}{\text { Initial wound area }}\right)(100)
$$

\section{Acute Dermal Irritancy Test}

OECD Guidelines were followed wherein furs in the dorsal area trunk of the New Zealand rabbits were shaved using a disposable and sanitized safety razor. A single dose of $0.5 \mathrm{~mL}$ preformulated hydrogel was applied to the shaved and intact skin using a $1 \mathrm{~mL}$ syringe, while the untreated skin areas served as the positive control. Treated areas were then covered with $6 \mathrm{~cm}^{2}$ sterile gauze pads and 1-inch micropore to hold the gauze pad in place. Three test patches were applied sequentially at different skin areas wherein patches were removed after three minutes, an hour, and 4 hours to observe and grade for any presence of severe skin reactions.

\section{Statistical Analysis}

Data collected was analyzed using One-Way Analysis of Variance (ANOVA) in Stata v.13 in which a p-value of less than 0.05 is considered significant.

\section{RESULTS}

\section{Extraction Yield}

The obtained dried methanolic leaf extract was $55.2 \mathrm{~g}$ (6.691\%) from macerated $825 \mathrm{~g}$ of $I$. coccinea dried leaves.

\section{Physicochemical Tests of the Extract}

Methanolic leaf extracts of $I$. coccinea was dark green with a leafy odor. It was slightly acidic with an average $\mathrm{pH}$ of 4.58 , soluble in water, sparingly soluble in ether, and practically insoluble with chloroform.

\section{Characterization of Preformulated Hydrogel}

As shown in Figure 1, the hydrogel base was transparent in appearance, smooth and homogenous in texture which 

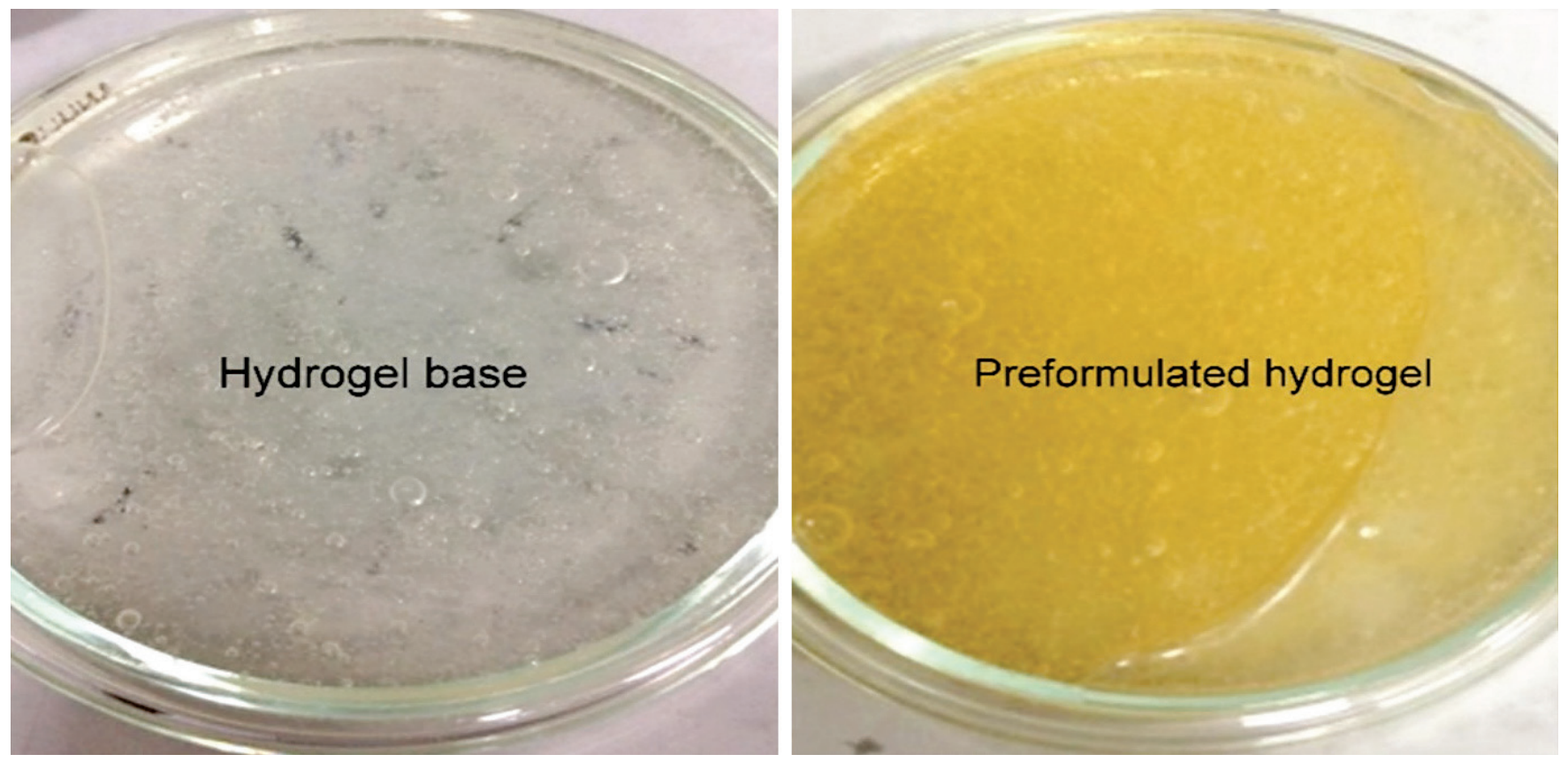

Figure 1. Hydrogel base appearance upon incorporation of $I$. coccinea leaf extract.

turned honey-colored with a sweet odor upon the addition of $I$. coccinea extract. Its average $\mathrm{pH}$ was 3.13 , viscosity 55.335 poise, and spreadability rate of 5.789. Also, it was sterile since turbidity was not observed on FTM and SCD after 14 days.

\section{In-vitro Antimicrobial Activity}

After a 24-hour incubation period, the antimicrobial activities of $2 \%$ mupirocin ointment (positive control), $2.5 \%$ preformulated hydrogel, and hydrogel base (negative control) were determined by measuring the inhibitory diameter zones against $S$. aureus, $S$. epidermidis and $P$. aeruginosa using a digital Vernier caliper. Based on the results shown in Table 4 below, the preformulated hydrogel had an average inhibitory diameter of $29.21 \mathrm{~mm}$ for $S$. aureus. Also, both preformulated hydrogel and hydrogel base were not effective against $S$. epidermidis and $P$. aeruginosa, unlike with $2 \%$ mupirocin ointment with average inhibitory diameters of $34.72 \mathrm{~mm}$, $32.91 \mathrm{~mm}$, and $22.50 \mathrm{~mm}$ on $S$. aureus, S. epidermidis and $P$. aeruginosa respectively. Results were statistically analyzed using One-way ANOVA wherein the $\mathrm{p}$-values acquired were $<0.05$ indicating a significant difference in the in-vitro antimicrobial activity against selected microorganisms.

\section{Pharmacologic Activity}

\section{Wound Healing Activity}

Wound contraction rates (WCR) of each treatment group were observed after $2^{\text {nd }}, 4^{\text {th }}, 8^{\text {th }}, 12^{\text {th, }}$ and $18^{\text {th }}$ day wherein the untreated group was the least on the $2^{\text {nd }}$ day $(\bar{x}=2.1)$, although it progressed after the $4^{\text {th }}$ day $(\bar{x}=19.73)$ and until the $12^{\text {th }}$ day of treatment $(\overline{\mathrm{x}}=97.12)$. These results were contrary to the group treated with preformulated hydrogel with WCR of $20.50 \%, 37.11 \%$ and $52.68 \%$ for $2^{\text {nd }}$, $4^{\text {th }}$, and $8^{\text {th }}$ day respectively, wherein complete healing were observed as early as $12^{\text {th }}$ day compared to the standard drug povidone-iodine with WCR of 4.93\%, 19.27\%, 31.86\%, $83.82 \%$ and $100 \%$ on the $16^{\text {th }}$ day. The group treated with hydrogel base also showed promising wound healing activity of $10.20 \%, 25.21 \%, 41.89 \%$, and $100 \%$ for $2^{\text {nd }}, 4^{\text {th }}$, $8^{\text {th }}$, and $12^{\text {th }}$ day respectively.

Table 4. Zone of Inhibition of Treatment Groups

\begin{tabular}{cccc} 
Treatment Groups & $\begin{array}{c}\text { S. aureus } \\
(\mathbf{m m})\end{array}$ & $\begin{array}{c}\text { S. epidermidis } \\
(\mathbf{m m})\end{array}$ & $\begin{array}{c}\text { P. aeurginosa }^{\mathbf{c}} \\
(\mathbf{m m})\end{array}$ \\
\hline $\begin{array}{c}\text { 2\% Mupirocin ointment } \\
(+) \text { control }\end{array}$ & $\begin{array}{c}34.72 \pm 2.13 \\
\text { Susceptible }\end{array}$ & $\begin{array}{c}32.91 \pm 0.153 \\
\text { Susceptible }\end{array}$ & $\begin{array}{c}22.50 \pm 0.435 \\
\text { Susceptible }\end{array}$ \\
\hline Preformulated hydrogel & $\begin{array}{c}29.22 \pm 0.229 \\
\text { Susceptible }\end{array}$ & $\begin{array}{c}0 \\
\text { Resistant }\end{array}$ & $\begin{array}{c}0 \\
\text { Resistant }\end{array}$ \\
\hline Hydrogel base & 0 & 0 & 0 \\
$(-)$ control & Resistant & Resistant & Resistant \\
\hline
\end{tabular}

Nb. $n=3 \times 3 ; p$-value ${ }^{a}=0.0273 ; p$-value $e^{b, c}=0.0221$ 


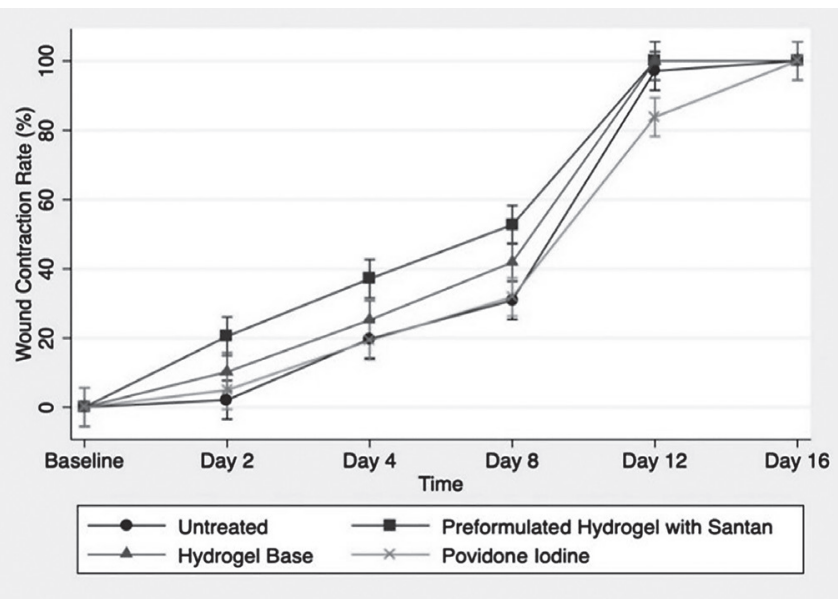

Figure 2. Mean \% wound contraction rate of the preformulated hydrogel and controls at different time periods.

As observed in Figure 2, there is no sufficient evidence to say that there is a significant difference in the mean wound contraction rate between untreated and povidone-iodine group $(p$-value $=0.730)$. This indicates that the povidoneiodine did not exert any wound healing property since its level of healing is similar to the untreated group. There is, however, a significant difference in the wound contraction rate between the untreated and povidone-iodine group and the preformulated hydrogel and hydrogel base. This indicates that both groups exerted wound healing property but the preformulated hydrogel is significantly higher than that of the hydrogel base ( $p$-value=0.006). The wound contraction rate significantly increases with time except from Day 12 to Day 16 ( $\mathrm{p}$-value=0.165) which showed no significant difference because most of the wounds have already healed on Day 12.

\section{Acute Dermal Irritancy Test}

As observed in Figure 3, the preformulated hydrogel topically applied to the test animals showed no signs of erythema, eschar, and edema. A confirmatory test was performed using a second rabbit (OECD, 2002) and still showed the same results wherein the skin was still intact when patches were removed indicating the non-irritancy of preformulated hydrogel.

\section{DISCUSSION}

The preformulated hydrogel containing $2.5 \%$ of methanolic leaf extracts of $I$. coccinea was honey-colored, sweet in odor, smooth, homogenous in texture, and acidic in nature with an average $\mathrm{pH}$ of 3.13. According to Nagoba et. al, wound healing progression decreases when $\mathrm{pH}$ is elevated to an alkaline condition. Also, an acidic environment helps in wound healing by controlling wound infection, altering protease activity, releasing oxygen, and enhancing epithelization and angiogenesis. ${ }^{14}$

Its viscosity and spreadability were 55.335 poise and $5.7887 \frac{\mathrm{g} * \mathrm{~cm}}{\mathrm{~s}}$ respectively in which gels containing CMC were noted for maximum spreadability range of 12.8 to 15 indicating greater spreadability of preformulated hydrogel. ${ }^{15}$ It was also sterile with no presence of turbidity after 14 days and upon comparison to FTM and SCD medias with inoculums of Bacillus subtilis and Candida albicans indicating good preformulation process. Since it was applied to wounded skins of test animals, sterile wound preparation
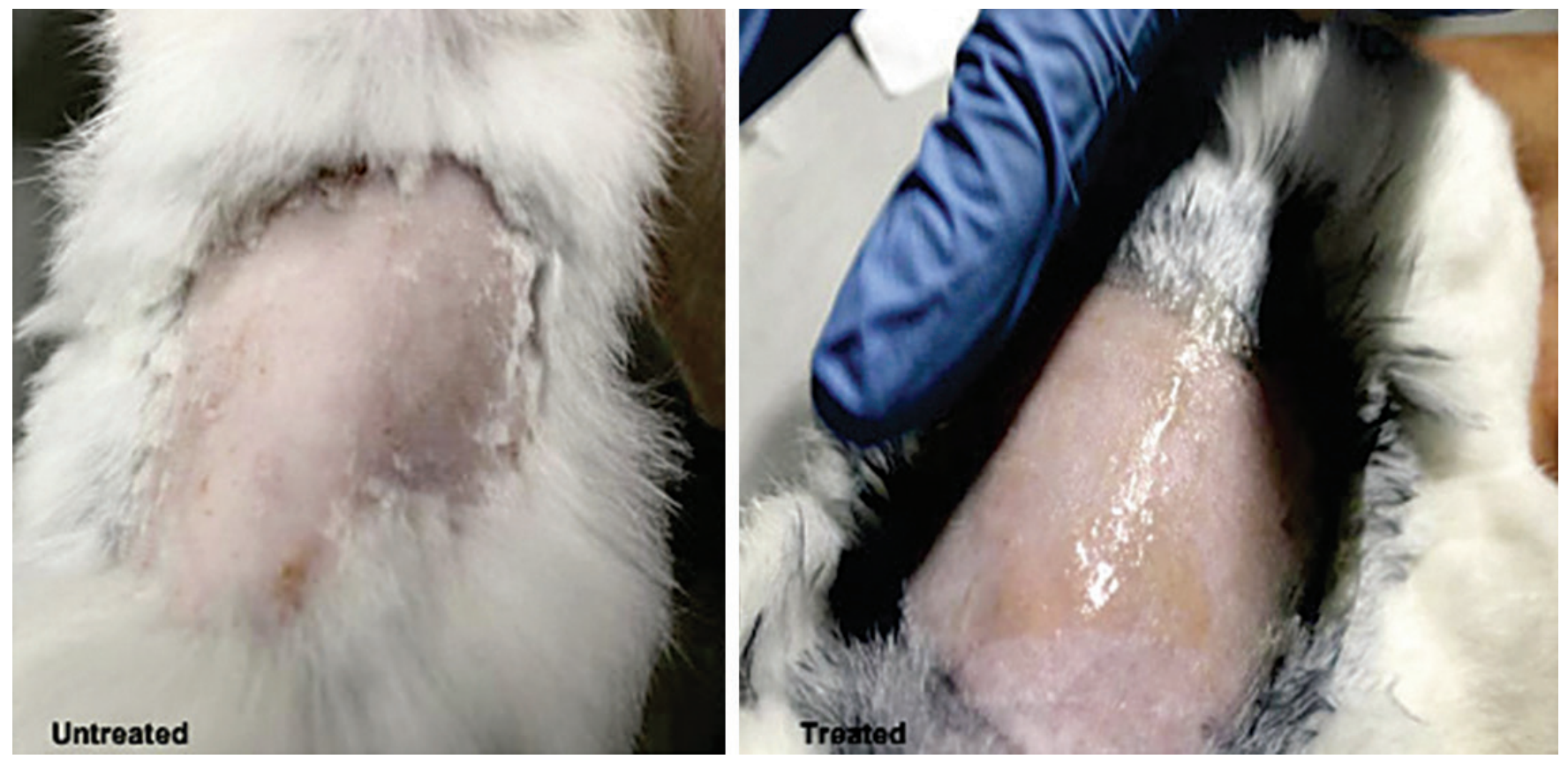

Figure 3. Comparison of untreated and treated patch testing. 
prevents further microbial contaminations that may impair the wound healing process. Also, the preformulated hydrogel was effective only to $S$. aureus with an average inhibitory zone of $29.22 \mathrm{~mm}$ and significantly lower to the standard drug 2\% mupirocin ointment. According to Marimuthu et. al, the antimicrobial activity of $I$. coccinea was due to its active constituents terpenoid, flavonoid, coumarin, alkaloid, and phenolic groups. ${ }^{16}$

Among the treatment groups, the preformulated hydrogel exhibited higher wound contraction rates which supported the previous studies of Chattopadhyay et. al on wound healing properties of the methanolic leaf extract with 96.78\% WCR higher than gentamicin sulfate (89.59\%) and non-treated control group. ${ }^{1}$ Hydrogel base alone exhibited wound healing activity thus supporting Shah and AminiNik (2017) findings that hydrogel dressings are superior to conventional dressings as they can maintain a moist environment that enhances wound healing. ${ }^{4}$ The speedy recovery of wounds observed in Group II and III can be due to CMC as a hydrogel base ingredient since it favored neutrophil accumulation at the wound site. Upon the addition of methanolic leaf extract, its wound healing activity was increased and made it more effective than the standard drug povidone-iodine $2.5 \%$. Also, the preformulated hydrogel was non-irritating with a Dermal Irritation Score of "0" as no presence of erythema, eschar, and edema were observed upon application. Histopathological observation and stability testing were delimited in this study and recommended to be determined in future research to fully assess its wound healing property and shelf-life.

\section{CONCLUSION}

The preformulated hydrogel containing $2.5 \%$ methanolic leaf extracts of $I$. coccinea was non-irritating, effective against $S$. aureus, has a better wound contraction rate, and a good substitute for povidone-iodine in wound treatment.

\section{Statement of Authorship}

All authors participated in data collection, analysis, and approved the final revision submitted.

\section{Author Disclosure}

All authors declared no conflicts of interest.

\section{REFERENCES}

1. Chattopadhyay P, Goyary D, Mazumder PM, Upadhyay A, Vijay V. Ixora coccinea Enhances Cutaneous Wound Healing by Upregulating the Expression of Collagen and Basic Fibroblast Growth Factor. ISRN Pharmacology. 2014 Jan; 751824.

2. Baliga, M. S., \& Kurian, PJ. Ixora coccinea Linn.: Traditional uses, phytochemistry and pharmacology. Chinese Journal of Integrative Medicine. 2012 Jan;18(1), 72-9.

3. Adnan, S. A., Al-Amin, M., Uddin, M. N., Shohel, M., Bhattacharjee, R., Hannan, J. M. A., \& Das, B. K. Analgesic, Anti-inflammatory and Antipyretic Effects of Ixora coccinea. Journal of Basic and Clinical Physiology and Pharmacology. 2014; 25(4), 423-8.

4. Shah, A.; Amini-Nik, S. The Role of Phytochemicals in the Inflammatory Phase of Wound Healing. International Journal of Molecular Sciences. 2017 May; 18(5), 1068

5. Dhivya S, Padma VV, Santhini E. Wound dressings - a review. Biomedicine . 2015 Dec;5(4):22.

6. Rescober, A. A. In Vitro Entrapment and Release Studies of Levofloxacin using Epichlorohydrin-Crosslinked Hydrogel. International Journal of Applied Pharmaceutics. 2019; 11(5): 138-44.

7. Maitra, J, \& Shukla, VK. Cross-linking in Hydrogels - A Review. American Journal of Polymer Science. 2014; 4(2), 25-31.

8. Capanema, N. S. V., Mansur, A. A. P., de Jesus, A. C., Carvalho, S, M., de Oliveira, L. C., \& Mansur, H. S. Superabsorbent crosslinked carboxymethyl cellulose-PEG hydrogels for potential wound dressing applications. International Journal of Biological Macromolecules. 2018 Jan;106, 1218-34.

9. Ramli, N. A. \& Wong, T. W. Sodium carboxymethylcellulose scaffolds and their physicochemical effects on partial thickness wound healing. International Journal of Pharmaceutics. 2011; 403(1-2), 73-82.

10. Clinical and Laboratory Standards Institute. Performance Standards for Antimicrobial Susceptibility Testing. 28th ed. CLSI supplement M100. 2018.

11. Jain N, Pathak R, Sandhu SS. Practices in Wound Healing Studies in Plants. Evidence-Based Complementary and Alternative Medicine. 2011 May; 438056

12. Moreira C. F., Cassini-Vieira P., da Silva, M. F. and Barcelos L. S Skin Wound Healing Model - Excisional Wounding and Assessment of Lesion Area. Bio-protocol. 2015 Nov; 5(22)

13. Chanabasappa Nilugal K, Fattepur S, Fadli Asmani M, Abdullah I, Vijendren, S., \& E, U. R. Evaluation of Wound Healing Activity of Swietenia macrophylla ( Meliaceae ) Seed Extract in Albino Rats. Journal of PharmTech Research. 2017 Aug; 7(5)

14. Nagoba BS, Suryawanshi NM,Wadher B, Selkar S. Acidic Environment and Wound Healing: A Review. WOUNDS. 2015 Jan; 27(1): 5-11

15. Metwally GF, Shukr MH. 2013. Evaluation of Topical Gel Bases Formulated with Various Essential Oils for Antibacterial Activity against Methicillin-Resistant Staphylococcus Aureus. Tropical Journal of Pharmaceutical Research. 2013 Dec;12(6):877-84

16. Marimuthu MM, Aruldass CA, Sandrasagaran UM, Mohamad S, Ramanathan S, et al. Antimicrobial activity and Phytochemical screening of various parts of Ixora coccinea. Journal of Medicinal Plant Research. 2014 Mar;8(10):423-9

\section{Funding Source}

No funding support. 